\title{
TOULOUSE FACE A LA GARONNE : EMPRISE DE L'URBANISATION DANS LA PLAINE INONDABLE ET GEOHISTOIRE DES AMENAGEMENTS FLUVIAUX
}

\author{
Philippe Valette \\ Maître de Conférences, Université de Toulouse Le Mirail, GEODE UMR 5602 CNRS \\ philippe.valette@univ-tlse2.fr \\ Jean-Michel Carozza \\ Maître de Conférences, Université de Strasbourg, GEODE UMR 5602 CNRS \\ carozza@equinoxe.u-strasbg.fr
}

\begin{abstract}
Résumé : La ville de Toulouse en France s'est installée sur les berges de la Garonne. L'évolution de l'urbanisation de la ville témoigne des relations ambivalentes qu'elle entretien avec le fleuve : fléau à combattre, contre lequel on aménage des digues, mais aussi ressource recherchée pour laquelle on crée des moulins, des ports... La géohistoire des aménagements fluviaux face à l'urbanisation révèle le développement et la juxtaposition des artéfacts sur le fleuve. Les paysages fluviaux urbains de Toulouse sont le résultat d'une lente construction temporelle. L'objectif de ce travail est de définir ces différentes temporalités, qui ont produit les paysages fluviaux actuels. Ces derniers font l'objet de projets de valorisation et leur mise en place permettra de changer les regards en mettant la Garonne au centre et au coeur de la ville.
\end{abstract}

Most-Clés : Toulouse, Garonne, urbanisation, aménagements fluviaux, ressource, inondation, géohistoire, rapport ville/fleuve, temporalités, paysages fluviaux.

\begin{abstract}
The city of Toulouse in France settled on the banks of the Garonne. The evolution of the urbanization of the city reflects the ambivalent relationship with the river: scourge to fight against dams which is landscape, but also sought to resource which is created mills, ports ... The
\end{abstract}

Recibido: 22-01-13. Aceptado: 8-10-13. 
historical geography of river developments facing urbanization reveals the development and juxtaposition of artifacts on the river. Urban river landscapes Toulouse are the result of a slow time construction. The objective of this work is to define these different temporalities that produced the current river landscapes. These are the subject of development projects and their implementation will change the looks by putting the Garonne in the center and heart of the city.

Key words: Toulouse, Garonne, urbanization, river engineering, resource, flood, geo-history, report city / river, temporality, river landscapes.

\section{Introduction}

Toulouse est indissociable de son fleuve, à tel point qu'aujourd'hui, les paysages de bords de Garonne sont devenus un des paysages identitaire de la ville. Ces paysages tant prisés de nos jours étaient totalement rejetés et désaffectés hier. Leurs physionomies actuelles sont le résultat d'une lente construction au cours du temps. Ils sont le fruit d'une forte volonté de maîtrise du fleuve par la société. Maîtriser la Garonne suppose de la part des sociétés riveraines de mettre en place des actions, des aménagements pour mieux exploiter son potentiel en ressource (eau, force hydraulique...), mais maitriser la Garonne signifie aussi de mettre en place des aménagements pour mieux s'en protéger.

Nous proposons ici d'étudier l'évolution du rapport entre la Garonne et la ville de Toulouse, selon une approche géohistorique et d'en faire une synthèse. L'objectif de ce travail est donc de déterminer les différentes temporalités qui ont produit les paysages fluviaux toulousains. L'approche géohistorique se base sur l'analyse de documents d'archives (textes, plans, cartes et photographies anciennes) situés dans différentes administrations (archives départementales et municipales). Ces documents anciens sont complétés par une recherche bibliographique la plus large possible. Les cartes et plans de la ville, mis bout à bout, permettent de connaitre l'emprise de la ville sur le fleuve et sa plaine inondable, depuis sa naissance. Nous traiterons de cet aspect dans la première partie. Dans une seconde partie, nous évoquerons les raisons du développement économique et urbain de la ville, qui doit beaucoup à l'exploitation de ses multiples ressources fluviales, et qui doit aussi s'affranchir des nombreuses crues et inondations de la Garonne. Enfin, dans la dernière partie, il sera question d'évoquer l'action des sociétés riveraines face au danger représenté par le fleuve au cours de l'histoire car les paysages fluviaux toulousains actuels sont le résultat de politiques de défenses face à l'aléa inondation. 


\section{La ville et son développement urbain face à la Garonne}

L'extension urbaine toulousaine dans la zone inondable s'est faite progressivement et il est possible d'identifier 5 périodes au cours de l'histoire.

\subsection{Une rive droite privilégiée au détriment des basses terres}

La question de la genèse de la ville de Toulouse est à l'origine de vifs débats entre historiens et archéologues. Aujourd'hui encore de nombreuses recherches sont menées pour répondre à cette interrogation (Pailler, 2002) et ont permis la tenue de nombreuses expositions où cette thématique est reprise (Bordes, 2005 ; Arramond, Requi, Vidal, 2004). Ou est située la ville Tolosa des Volques Tectosages avant la ville actuelle? A cette époque, Tolosa se concentre autour de plusieurs noyaux différents, où Vielle Toulouse (150 ha dominant la Garonne près du confluent avec l'Ariège) et St Roch (80 ha dont le développement serait lié au commerce sur le fleuve) jouent un rôle majeur (cf figure 1). "Malgré sa taille et son importance politique et économique, l'agglomération gauloise n'a laissé aucune trace perceptible dans le paysage actuel de Toulouse (...)" (Vaginay, 2005).

Si la filiation avec les sites protohistoriques précédents est difficile à établir, elle n'est pas à l'origine de la ville actuelle qui est fondée deux siècles avant notre ère par l'Empire romain dans le cadre de la conquête militaire du Midi de la Gaule (la Narbonnaise). "C'est en fait la fondation de la capitale romaine des Tectosages qui va marquer de son empreinte tout l'avenir de l'urbanisme toulousain jusqu'à aujourd'hui " (Vaginay, 2005).

Sans tomber dans un déterminisme strict, le choix d'implantation de la ville romaine au Nord du site de St Roch, semble s'inscrire pour partie dans une logique de site. La coïncidence entre le site d'implantation et la rupture dans la dynamique fluviale de la Garonne n'y est peut-être pas étrangère. Jusque-là, la Garonne inonde sa rive gauche, qui est la rive basse. En aval, l'infléchissement du tracé suivant une direction SW-NE renverse cette dynamique, la rive droite devenant la rive basse inondable.

A cet endroit le fleuve décrit une boucle qui vient butter contre un lambeau de terrasse sur la rive droite, encadré par les buttes de Pech David et de Calvinet. Ainsi, la terrasse de rive droite élevée à $15 \mathrm{~m}$ au-dessus du niveau de la Garonne se trouve à l'abri des inondations. La rive gauche, par contre, est inhospitalière et est fréquemment inondée. Cette situation, à l'abri des crues, est aussi favorisée par la possibilité de traverser la Garonne à travers la présence d'un ou plusieurs gués : démontré pour le Bazacle et supposé pour la Daurade. Mais, comme le souligne Labrousse (1968), le choix du site est également le résultat de la combinaison de facteurs à plus petite échelle. 


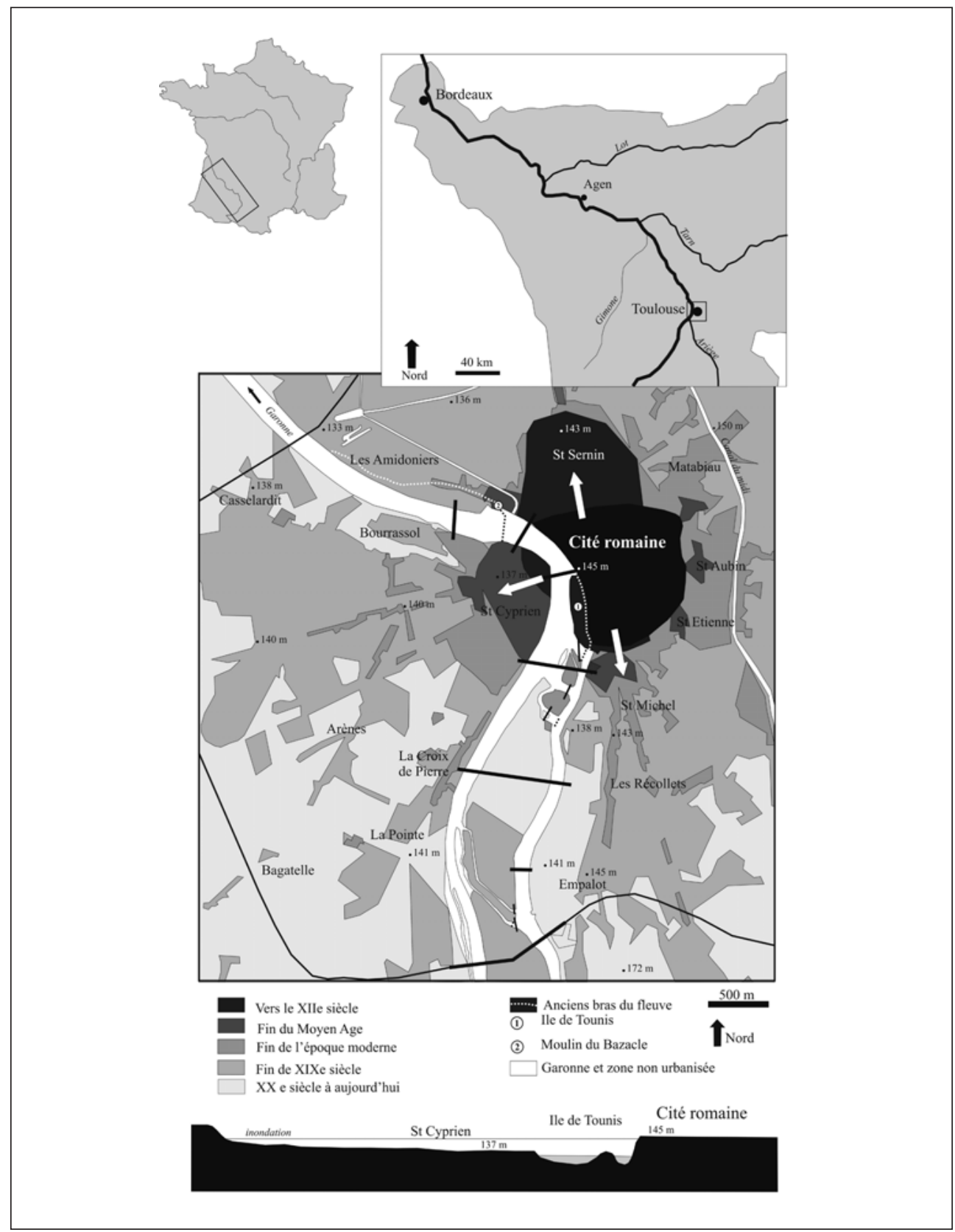

Figure 1. Extension de I'urbanisation de Toulouse face a la Garonne. Source: Archives municipales. À partir de la comparaison de plans anciens. 
"Trois faits géographiques commandent le site de Toulouse et ont conditionné la fortune de la ville : la Garonne et ses gués, la terrasse de 15 mètres où fut bâtie la ville romaine, la trouée de Montaudran"(Wolff, 1974). Ces trois facteurs ont engendré l'installation d'un noyau urbain. D'ailleurs dès le départ cette ville apparaît comme un lieu de jonction d'une rive à l'autre. Dans le toponyme "tolosa " la syllabe tol désigne un gué sur une rivière. Mais c'est aussi un carrefour commercial entre le Languedoc et la Gascogne. La vallée de l'Hers, que l'on rejoint par "la trouée de Montaudran " conduit vers la Méditerranée et la vallée de la Garonne vers les Pyrénées et l'Océan Atlantique. L'époque romaine tirera de nombreux avantages de cette situation de carrefour, en utilisant notamment l'axe garonnais comme artère de navigation. Au IIe siècle après notre ère, on dénombre en moyenne 20000 habitants vivant à l'intérieur d'un vaste territoire de 90 hectares (Labrousse, 1968).

Sur les rapports de la ville au fleuve pour cette période, les données historiques et archéologiques sont peu loquaces. Seule la présence d'un bâtiment dont l'interprétation est encore mal comprise au niveau du Bazacle (gué ?) pour la période antique atteste d'une ville déjà en partie tournée vers le fleuve.

\subsection{Une tentative de conquête des basses terres (la ville médiévale)}

La ville romaine reste cantonnée sur la rive droite de la Garonne et délaisse les parties basses de la vallée. Par contre les ressources notamment en eau de la rive gauche sont exploitées comme l'atteste l'aqueduc antique qui restera une source d'alimentation en eau de la ville jusqu'à la période Moderne. A partir de ce site romain, la ville médiévale va s'étendre à la fois au Nord, au Sud et à l'Ouest et se partage en trois entités spatiales bien distinctes : la Cité, le Bourg et les faubourgs. La Cité, ancienne ville antique, se développe et un Bourg apparaît progressivement au cours du Moyen-Age central au Nord, dans le prolongement de la terrasse alluviale. Il se cristallisant sur les abords " du cimetière paléochrétien établi autour du monastère suburbain de Saint-Sernin et de sa basilique funéraire " (Macé, 2005). Deux faubourgs sont à distinguer durant cette période : St Michel et St Cyprien. Au XIIe siècle, une salvetat est créée dans les environs du faubourg St Michel. Elle est située hors des murs dans les environs du château Narbonnais (Chalande, 1919). Cette salvetat est lotie pour permettre l'installation de futurs habitants venant du Lauragais. Une deuxième salvetat est aussi créée au XIIe siècle, le long de la Garonne non loin du château Narbonnais et correspondrait à l'île de Tounis, située sur la rive droite (Tourrilhes, 1987). Elle est occupée à partir de la fin du XIIIe siècle par des personnes vivant des métiers liés à la Garonne. "De la naissance à la mort, du travail aux loisirs, de la richesse à la misère, c'est l'eau qui guide la vie entière des tounisiens "(Arrouy, 2005). L'île de Tounis est un quartier occupé, dès le départ, par les basses couches sociales On pourrait comparer la situation de l'île de Tounis avec celle du Bazacle, moulin construit au XIIe siècle, situé hors de la ville sur la rive droite en 
zone inondable, mais que l'on s'empressera de fortifier pour le protéger et l'intégrer dans la ville médiévale. A l'Ouest de la ville, hors des murs se développe à la même époque le faubourg St Cyprien, sur les routes de Gascogne et de Comminges (Macé, 2005). St Cyprien s'organise autour de l'église St-Nicolas, patron des mariniers et il est également invoqué dans toute la vallée de la Garonne par tous ceux qui sont exposés aux crues et aux inondations (St Nicolas de la grave, St Nicolas de Balerme...). Le faubourg St Cyprien est implanté en zone inondable sur la rive gauche et il a fait face, au cours de l'histoire, à l'envahissement fréquent des eaux de la Garonne.

Au XIVe siècle, "Toulouse compte alors une population d'environ 35000 habitants ce qui en fait une des plus importantes cités du royaume "(Bordes, 2005). C'est aussi durant cette période que la ville est enclose de remparts, qu'elle gardera jusque vers le début du XIXe siècle. A l'intérieur de la ville médiévale, le rapport avec la Garonne s'intensifie autour des activités commerciales (navigation) et proto-industrielles (moulins). C'est aussi durant cette période que la dissymétrie géomorphologique du site se double d'une dissymétrie sociale: la rive droite à l'abri des inondations abrite la ville des élites alors que la rive gauche, soumise aux inondations accueille les activités indésirables. Sur la rive droite, dans la Cité et le Bourg se localisent les établissements civils publics, universitaires, religieux, des commerçants, des artisans, des habitations et en périphérie des quartiers ruraux, soit la majeure partie de la population et des activités économiques (Marconis, 1996). La rive gauche n'est occupée que par le petit faubourg de St Cyprien, rassemblant les activités ostracisée comme les hôpitaux, les léproseries, les abattoirs et où se concentre les populations marginales. L'espace habité du quartier St Cyprien à l'époque médiévale est peu dense et essentiellement occupé par des territoires ruraux. L'île de Tounis située sur la rive droite appartient également à ce monde. Le plan de Nicolas Bertrand daté de 1515 confirme cette dissymétrie topographique et sociale (Archives Municipales de Toulouse, II 699). L'auteur a représenté les monuments les plus caractéristiques de la rive droite constituant un véritable panorama d'églises et château (Château Narbonnais, cathédrale Saint-Etienne, Saint-Sernin...). En face, le quartier St Cyprien semble moins pourvu de bâtiments et l'île de Tounis, en position centrale par rapport à la Garonne paraît se rapprocher du quartier " des indésirables " (cf figure 2).

\subsection{Un gonflement des faubourgs de part et d'autre du fleuve (Moyen Age jusqu'à la fin du XVIIle siècle)}

L'extension urbaine médiévale n'évolue pratiquement pas jusqu'au XVIII siècle. "Dans son enceinte de murs et de tours telle que nous la restituent les plans de 1631 et 1678, Toulouse n'apparaît guère différente de ce qu'elle était au Moyen-Age " (CUIET, 1993). Le célèbre plan de Toulouse de Melchior Tavernier en 1631 confirme cet état et montre bien la situation de la ville au XVIIe siècle, où les zones vides médiévales à l'in- 


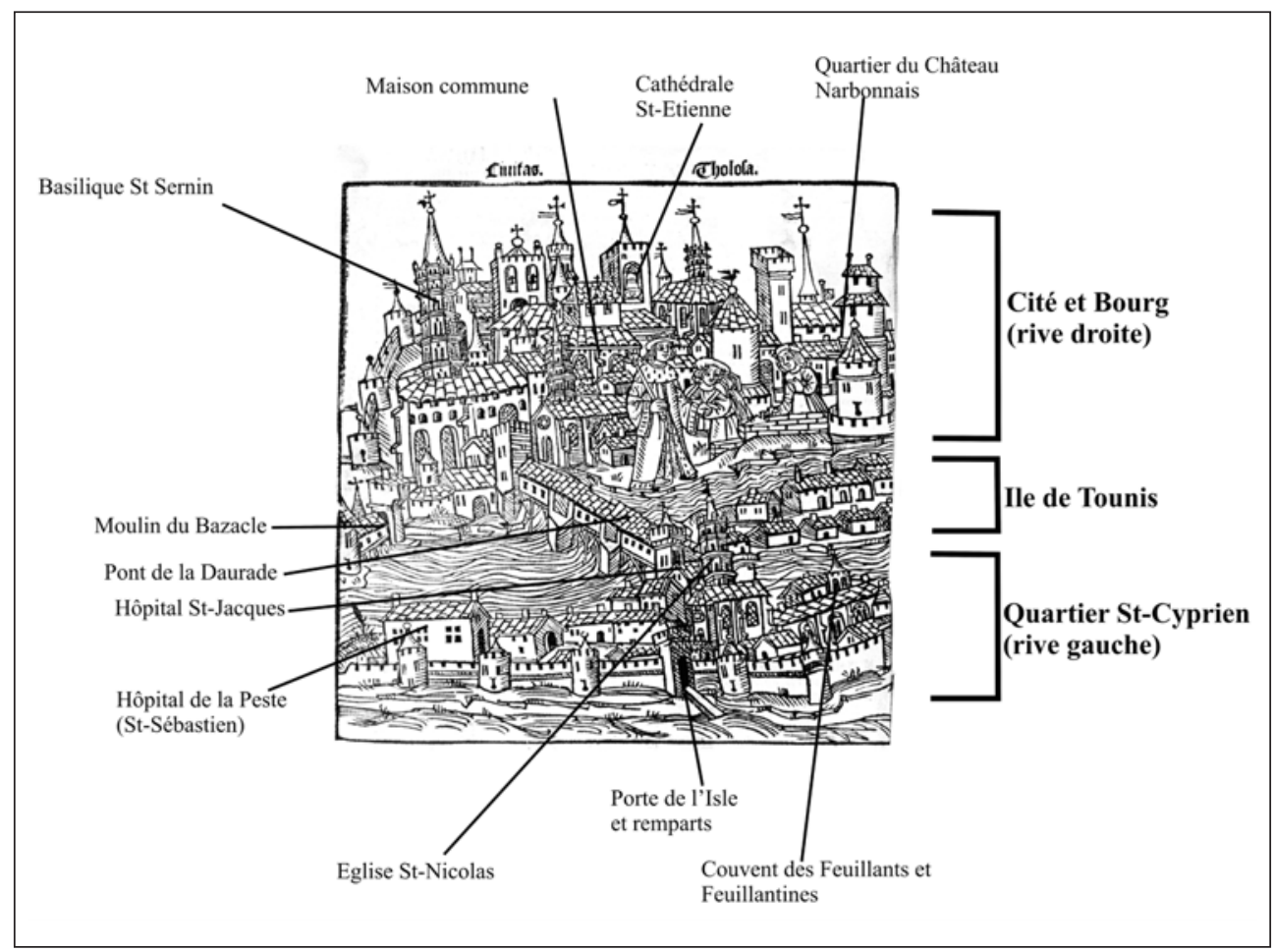

Figure 2. Vue perspective de Toulouse en 1515.

Source: Gravure sur bois, Nicolas Bertrand, Archives Municpales de Toulouse, II 699, 1515.

térieur des remparts se remplissent peu à peu (cf figure 3). Sur ce plan, on retrouve les deux excroissances de la rive droite correspondant à la Cité romaine et au Bourg médiéval. Le quartier St Cyprien est entouré également de remparts et de fossés lui donnant un caractère d'île. D'ailleurs, à cette époque, on entre dans St Cyprien par la porte de l'Isle. Le plan de 1678 d'Albert Jouvin de Rochefort, plus tardif, montre une ville semblable à celle de 1631, mais une extension du faubourg St Cyprien situé hors de la ville et hors des remparts. Deux faubourgs embryonnaires font leur apparition en rive droite : le faubourg St Etienne et le faubourg Matabiau. Au XVIIIe siècle, l'expansion urbaine se confirme et "le dynamisme économique s'accompagne d'un vif essor démographique, qui porte la population de la ville à plus de 60000 habitants en 1789 " (Taillefer, 2005). Avec cette expansion démographique plusieurs faubourgs se développent comme les faubourgs St Aubin, St Sauveur, St Etienne, St Michel et St Cyprien. Malgré cette expansion urbaine la densité urbaine reste déséquilibrée : 320 habitants à l'hectare dans la Cité, 120 habitants à l'hectare dans le bourg et seulement 65 à St Cyprien (Taillefer, 2005). Malgré tout, cette extension du faubourg St Cyprien semble montrer 


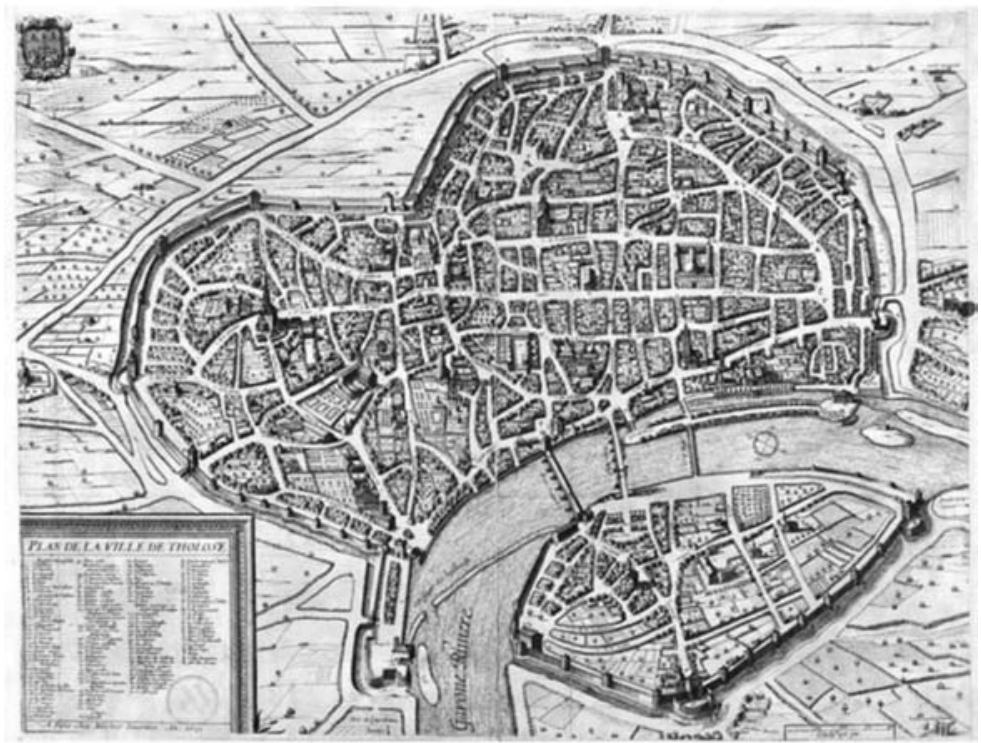

Plan Melchior Tavernier, 1631 (Archives Municipales de Toulouse, 20 Fi 359).

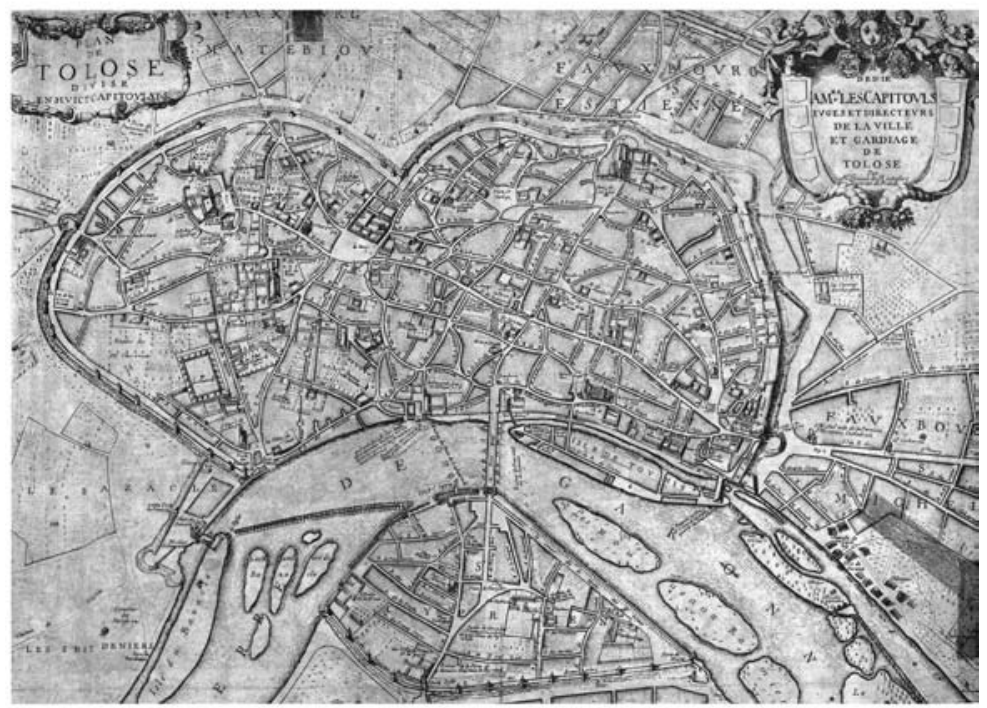

Plan Jouvin de Rochefort, 1678 (Musée Paul Dupuy, Inv 1.341).

Figure 3. La ville de Toulouse au XVIle siècle. 
une société urbaine s'affranchissant progressivement de la contrainte des inondations. L'expansion démographique de la ville de Toulouse doit beaucoup à la modernisation de l'agriculture et surtout à l'ouverture du Canal des Deux-mers en 1681 permettant de relier Méditerranée et Atlantique par voie d'eau.

\subsection{La colonisation des îles de la Garonne, une ville pré-industrielle (XIXe siècle jusqu'à la moitié du XXe siècle)}

A partir du XIXe siècle, les nouveaux faubourgs du XVIIIe siècle s'intensifient et multiplient, de toutes parts, la ville par trois, y compris en rive gauche, pourtant complètement submergée par la crue de 1875. Ces faubourgs, à l'extérieur des remparts, remplacés par des boulevards vers 1830, perdent progressivement durant cette période leur caractère semi-rural pour devenir des quartiers urbains composés de modestes villas (appelées " toulousaines "). Les boulevards jouent alors un rôle de lien entre les anciens quartiers et les faubourgs. En 1886, la ville atteint 150000 habitants et 175000 en 1926 (Peyrusse, 2005).

Si certaines îles de la Garonne sont investies par des activités pré-industrielles dès le XVIIIe siècle (et même avant pour certaines, durant la période médiévale), c'est surtout au XIXe siècle qu'elles se développent grandement en créant de véritables quartiers préindustriels utilisant la force hydraulique du fleuve (île d'Empalot, du Ramier, de Tounis et du Bazacle). Parmi ces îles, la plus anciennement occupée dès le Moyen-Age est lîle de Tounis. Cette île est vouée à un artisanat pré-industriel avec une diversité de corps de métiers représentés (textile, alimentation, restauration, bois, construction de bâtiments, tanneries, métaux, verrerie, papeterie). Cette île située en amont du pont Neuf est une source de pollution (abattoirs, tanneries...) pour l'eau de la Garonne dans la ville entière. Plus en amont, l'île du Ramier est investie par un moulin à poudre dès 1675 (Loncan, 1998), activité déplacée en 1848 au sud de l'île " pour assurer la sécurité des habitants sans cesse menacée par des explosions destructrices " (Armengaud, Vieu, 1998). La première extension de la poudrerie a lieu en 1877 sur un terrain de 3 ha, plus au Sud, sur le ramier d'Empalot. La seconde extension de la poudrerie s'opère au début du XXe siècle en 1914, sur la partie Sud de l'île d'Empalot à laquelle on ajoutera un complexe industriel sur le site de Braqueville, rive gauche. Dans ce quartier industriel, l'Office National et Industriel de l'Azote (ONIA devenue plus tard AZF) s'implante en 1921 pour produire de l'engrais, dont on connaît la triste destiné aujourd'hui (explosion AZF de 2001).

C'est aussi durant cette première moitié du XXe siècle que l'île du Ramier, après avoir déménagé la poudrerie, connaitt une urbanisation liée aux loisirs, avec le parc toulousain (espace vert disparu aujourd'hui) et le parc des sports (piscine d'été, stadium...) créé dans les années 1930 (Armengaud, Vieu, 1998). Ailleurs, l'activité préindustrielle et industrielle s'étend en aval de la ville, où à la fin du XIXe siècle le complexe du Bazacle 
regroupe 16 établissements industriels diversifiés (filature, manufacture des tabacs, tannerie...) alors que la vue panoramique de Colignon en 1642 nous montre le seul moulin du Bazacle fortifié (cf figure 4). Ce quartier porte le nom évocateur des Amidoniers. Il est situé en aval du Bazacle, entre le canal de Brienne et le bras secondaire de la Garonne et il s'urbanise progressivement jusqu'à la première moitié du XXe siècle.

Si entre le XIXe siècle et la première moitié du XXe siècle, la ville s'urbanise progressivement, elle s'industrialise également. Cette industrialisation, au départ localisée en dehors de la ville, est progressivement englobée à la ville entre la seconde moitié du XXe siècle et aujourd'hui.

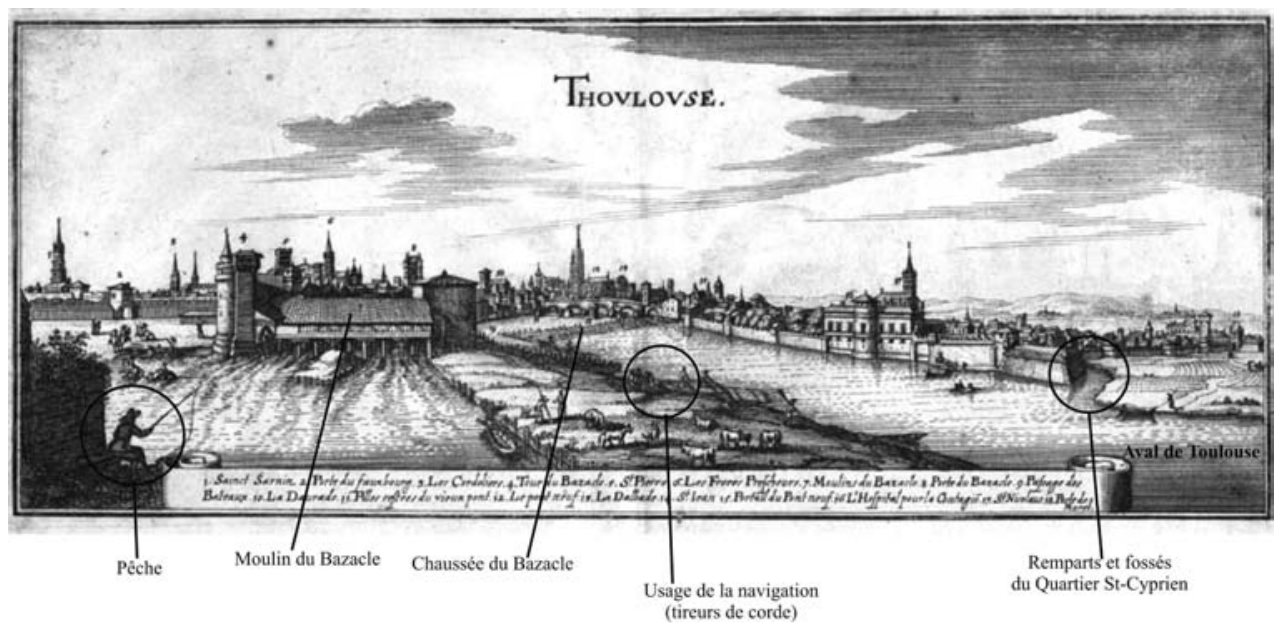

Figure 4. Vue particulière de Toloze en 1642.

Colignon, Musée Paul Dupuy.

\subsection{L'explosion urbaine de la seconde moitié du XXe siècle}

"Dans les années 1945-1955, la ville fait encore figure de "grand village " (Marconis, 1996). Depuis la seconde moitié du XXe siècle, Toulouse a connu une explosion démographique et urbaine recouvrant d'artefacts les territoires de part et d'autre de la Garonne. Au 1 ${ }^{\text {er }}$ janvier 2007, 439453 habitants sont comptabilisés dans la commune toulousaine, l'agglomération (58 communes) regroupe 859111 habitants et l'aire urbaine 1118476 habitants. La ville s'étale largement sur la rive gauche en zone inondable et déborde le quartier St Cyprien, jusqu'à s'étendre sur la terrasse alluviale, où les anciens villages sont aujourd'hui des cités de banlieues (Tournefeuille, Colomiers...). Sur la rive droite, l'urbanisation s'étend jusque dans la vallée de l'Hers et même 
au-delà. Les berges de la Garonne et la plupart des îles sont également urbanisées. Seules des parties de zones inondables en rive droite au Nord (quartier de Ginestous) et au Sud sur d'anciennes îles ne sont pas encore densément urbanisées, jusqu'à quand ?

Le développement urbain de la ville de Toulouse, au cours de l'histoire, a largement colonisé la plaine inondable de la Garonne (cf figure 1). Ce développement dans la plaine inondable est lié à l'exploitation de multiples ressources et il a, également, longtemps été limité par les désastres provoqués par les crues et les inondations du fleuve.

\section{Le fleuve dans la ville, la Garonne à Toulouse}

\subsection{L'exploitation de la force hydraulique... au cœur de l'économie}

Les aménités du fleuve constituent une motivation dans l'appropriation de ces espaces de berge, de plaine inondable et de terrasse. Parmi ces avantages ou ces ressources, la puissance hydraulique des eaux courantes joue un grand rôle à travers l'aménagement de moulins. La puissance hydraulique est aussi exploitée à travers l'usage de la navigation fluviale.

La ville de Toulouse doit une partie de son développement économique et démographique aux différentes cultures produites dans les campagnes (blé, pastel...). La production agricole acheminée à Toulouse est ensuite " traitée " dans des moulins qui utilisent la force hydraulique du fleuve durant la période médiévale. "La Garonne se présentait comme une réserve presqu'illimitée de force motrice " (Tourrilhes, 1987). Ces établissements industriels permettent au départ de moudre des céréales. Ils seront aussi utilisés pour d'autres activités (fabriquer du tissu, du papier, battre le fer...). Le premier texte qui mentionne des moulins à Toulouse date de la fin du XIe siècle, entre 1071 et 1080 (charte de fondation de l'hôpital St Raymond). Il s'agit de moulins à nef, c'est-à-dire de moulins flottants amarrés sur la Garonne et qui profitent des chenaux où le courant de la Garonne est le plus fort. Ce type de moulins est très fréquent sur la Garonne, jusqu'au XVIIIe siècle et de nombreuses cartes anciennes en font mention. "Toulouse en a possédé un grand nombre au moins une soixantaine au total " (Mercié, 2007). Ces moulins à nefs étaient essentiellement concentrés sur trois sites : le Bazacle, la Daurade et le Château Narbonnais. A partir de la fin du XIIe siècle, des moulins terriers sont aménagés sur les berges comme en 1192 pour le moulin du Château Narbonnais et 1194 pour le moulin du Bazacle et une date voisine pour le moulin de la Daurade (cf figure 5). Ce dernier a disparu dans la seconde moitié du XIVe siècle. Par contre, ceux du Bazacle et du Château Narbonnais sont à l'origine de véritables quartiers préindustriels. Il faut ajouter à ces moulins d'autres moulins qui se sont établis sur les berges de la Garonne plus tard. 


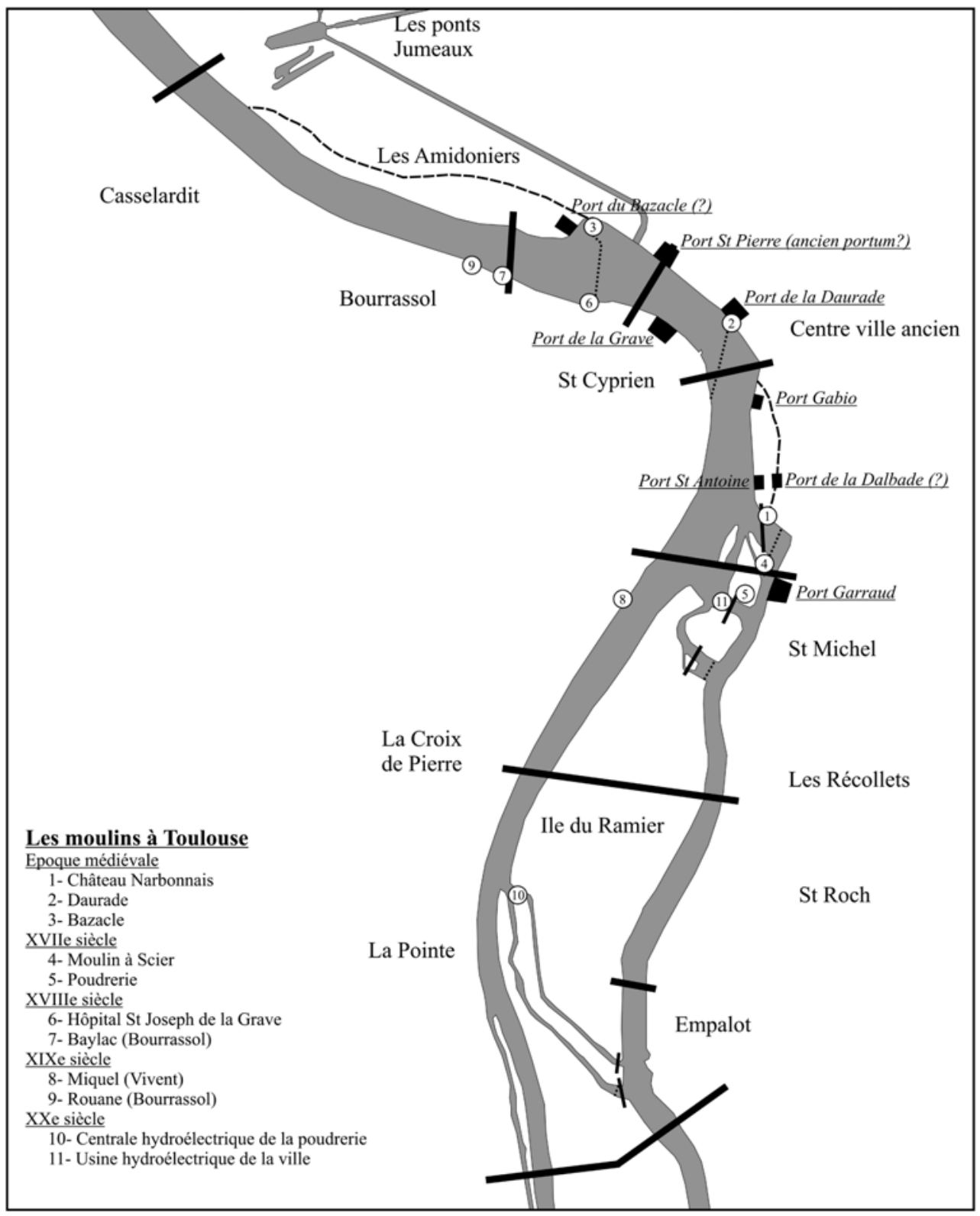

Figure 5. Les moulins, chaussées et ports fluviaux de Toulouse. 
Le plan de Jouvin de Rochefort daté de 1678 montre dans les environs du quartier St Michel deux moulins : un moulin à scier situé en amont du moulin du Château Narbonnais sur une île et un moulin à poudre face au port Garraud. Entre la fin du XVIIe et le début du XVIIIe siècle, le moulin de l'Hôpital St Joseph de la Grave est alimenté en force hydraulique par la Garonne et on avait aussi imaginé de tirer profit des eaux qui s'écoulent des fossés de la ville (Mercié, 2007). Dans le quartier de Bourrasol, sur la rive gauche plus en aval, un autre moulin est construit vers la fin du XVIIIe siècle (cf figure 5). Il ne reste quasiment plus de traces matérielles de ce moulin dans le paysage fluvial urbain aujourd'hui. Au début du XIXe siècle, plusieurs moulins sont également construits comme le moulin Miquel ou Vivent établi non loin de la porte de Muret en amont de la ville. Dans les années 1830, un autre moulin est construit dans le quartier Bourrasol, le moulin du château d'eau Rouane. Il est alimenté en eau par un canal venant des eaux d'évacuation du château d'eau.

Tous ces moulins, dont certains ont disparu, produisent de l'électricité depuis la fin du XIXe siècle.

La navigation fluviale apparaît comme une autre source de richesse pour la ville de Toulouse. La ville reçoit des marchandises des territoires pyrénéens, méditerranéens par la Voie Narbonnaise puis par le canal du Midi à partir du XVIIe siècle et est relié à l'Atlantique par la Garonne. La Garonne apparaît comme un axe commercial de grande importance où transitent denrées agricoles, bois, roches, personnes..., ce qui se traduit par la présence de nombreux ports dans la ville. Aujourd'hui, le ou les ports antiques ne sont pas connus. Par contre, plusieurs sites sont identifiés comme ports fluviaux toulousains durant la période médiévale. Le port Garaud est mentionné au XIVe siècle dans les textes et certains auteurs lui donnent une origine au XIIe siècle (Coppolani, 1951). Il se situait à l'entrée Sud de la ville sur la rive droite dans les environs du quartier St Michel. Sur l'île de Tounis, le port médiéval St Antoine est situé sur la façade Ouest de l'île au carrefour du pont de Comminges et la Garonne. Il change de nom et devient le port de la Roquette au XVe siècle. Au XIIIe siècle, un autre port est mentionné entre l'île de Tounis et la garonnette, obligeant les bateaux à remonter le bras secondaire (cf figure 5). Il s'agit du port de la Dalbade dont on sait assez peu de choses, mais qui semble se situer "près de la route, hors les murs de la ville jusqu'à la Garonne " (Dubourg, 1883). Plus en aval, le port de la Daurade (appellation apparue au XIVe siècle) est rattaché au monastère du même nom, qui percevait par la même des taxes. Ce port s'appelait Portus Vivarias dans les actes du XIIe siècle. Un peu plus au nord deux ports sont mentionnés dans les textes médiévaux. Il s'agit du portum associé à St Pierre des cuisines, qui apparaît en 1335 et du port du Bazacle mentionné en 1339 (Tourrilhes, 1987). Enfin, un dernier port situé sur la rive gauche apparaît en 1440, vraisemblablement avant, le port de la Grave associé au quartier St Cyprien. L'analyse du plan Jouvin de Rochefort en 1678 (cf figure 3) rajoute un port supplémentaire, le port de la Gabio situé dans les environs de l'île de Tounis (Fortuné, 1988). Parmi tous ces ports, quatre subsistent jusqu'à la période du déclin de la navigation sur la Garonne, vers la 
fin du XIXe siècle (port Garraud, St Cyprien, Daurade, et Vidou situé dans les environs du portum médiéval et port St Pierre aujourd'hui).

S'il reste encore beaucoup d'interrogations sur la localisation précise de certains ports, la diversité des mentions témoignent d'une activité économique de grande ampleur sur les berges de la Garonne à Toulouse.

\subsection{Une eau de boisson indispensable aux toulousains et un espace de loisirs}

La Garonne est aussi une ressource indispensable pour les toulousains notamment parce qu'elle permet l'alimentation en eau potable de la ville. Si l'eau de la Garonne est utilisée pour l'industrie et les transports, elle est aussi une " ressource naturelle mise à contribution pour l'alimentation, l'hygiène, les loisirs (...) "(Pelletier, 1990). On boit, on se lave et on lave son linge dans l'eau de la Garonne, ce qui n'est pas sans poser des problèmes de salubrité. La Garonne est à la fois un aqueduc alimentant en eau potable la ville et un cloaque, où on rejette ses immondices. Durant la période moderne, "Tounis offrait un état sanitaire particulièrement déplorable ; on y rencontrait sans cesse des maisons dépourvues de latrines, empuanties par des matières fécales, des puits aux eaux contaminées par les infiltrations, des étables de porcs voisinant avec les pièces habitées..." (Cathala, 1962). Cette situation particulière à l'île de Tounis se rencontre ailleurs sur les berges du fleuve. D'ailleurs l'alimentation en eau potable a été pendant longtemps le grand problème de la ville de Toulouse, au regard des souillures de la rivière (Laffont, 1997). Au début du XIXe siècle la construction de château d'eau, dont le but est de distribuer l'eau à 60000 toulousains, après sa captation dans la Garonne et son épuration dans la prairie des Filtres, règle le problème pour un temps. La Garonne permet une autre forme d'alimentation par l'intermédiaire de la pêche, source de nourriture pour les toulousains. On sait assez peu de choses sur cet usage, mais certaines représentations de la ville témoignent de cette pratique, comme la représentation de François Colignon en 1642 (cf figure 4).

Le fleuve et ses abords immédiats est également un espace de loisirs où on organise des fêtes nautiques, des courses de chevaux, des corridas, des rencontres sportives... Du reste l'île du ramier est vouée en grande partie à cet usage puisque dans la première moitié du XXe siècle des équipements sportifs (piscines, stades...) viennent remplacer le parc toulousain, ancien espace vert de la ville voué à la promenade.

Si le fleuve garonnais est à l'origine de ressources diverses, il est aussi un fléau, à travers ses crues et inondations. 


\subsection{Les crues et les inondations de la Garonne, un fléau pour la ville}

La thématique des crues et des inondations de la Garonne, et en particulier à Toulouse a fait l'objet de nombreuses publications. "Enumérer la longue litanie des aïguats de la Garonne serait fastidieux ; d'autres l'ont fait "(SMEPAG, 1989). La plus importante chronologie des crues a été réalisée par Jules Serret en 1900. Il est possible de la compléter par les travaux de Lafaille (1687), Chalande (1912), Lambert (1982 ; SMEPAG, 1989). Malgré tous ces travaux, il est difficile de classer et de comparer les crues entre elles. Quand bien même les hauteurs d'eau sont conservées, elles ne sont mesurées qu'à partir du XIXe siècle et fiables à partir de 1880. Avant ces dates, les indications de hauteur d'eau sont évoquées en fonction des "lunes " d'ouverture des piles du pont neuf, atteintes ou pas. Les indications sont donc majoritairement descriptives par rapport aux dégâts et aux dommages causés. Certaines inondations ont fait l'objet de nombreuses publications monographiques comme celles de 1875, qui sert d'inondation de référence aujourd'hui. Enfin, face au fléau des inondations garonnaises, de nombreux auteurs définissent ou esquissent des projets de protection, dont certains ne seront pas suivis d'effets. Parmi eux, sans être exhaustif, on peut citer : Payen en 1867, Vidalot en 1884, Pendariès en 1917, Saint en 1918, Morel en 1922 et dernièrement Lambert (SMEPAG, 1989).

Dans les environs de Toulouse, la Garonne possède un module voisinant les $200 \mathrm{~m}^{3} / \mathrm{s}$ pour un bassin versant d'environ $10000 \mathrm{~km}^{2}$. La caractéristique principale de ce fleuve est son caractère excessif alternant fortes crues, inondations et étiages sévères, les extremums s'échelonnant entre $8000 \mathrm{~m}^{3} / \mathrm{s}$ (débit estimé de l'inondation de 1875) et $20 \mathrm{~m}^{3} / \mathrm{s}$ en étiage. Cependant, ce sont surtout l'importance et la multiplicité des crues et inondations qui caractérisent mieux la Garonne. Le bassin versant de la Garonne de par sa position géographique, reçoit une partie des eaux tombant sur les Pyrénées et la Garonne y est une rivière de piémont à régime pluvionival. L'encaissement relatif du lit à pleins bords s'atténue de plus en plus à l'approche de la ville. Or dans le même temps, le lit " ne s'élargit pas assez, compte tenu de la réduction de la pente de la rivière, pour permettre à lui seul le passage des débits de crue "(Lambert, 1982). Dans cette configuration, le lit majeur évacue fréquemment le surplus d'eau ne pouvant pas s'écouler dans le lit à pleins bords. La cartographie du risque identifie plusieurs enveloppes d'inondation à Toulouse, déterminées par la fréquence des événements. Ainsi, les parties les plus proches du fleuve dans la ville, composées essentiellement d'îles (actuelles et/ou anciennes) correspondent aux crues très fréquentes et fréquentes. Le reste c'est-à-dire le lit majeur peut se définir comme le lit d'inondation exceptionnelle (cf figure 6).

\subsection{La chronique des crues à Toulouse}

L'aïguat (terme languedocien signifiant inondation, parfois orthographié ayguat) est perçu comme un fléau par les toulousains car il peut provoquer de multiples désagréments. Une crue provoque des érosions de berge, ce qui semble fréquent à Tou- 


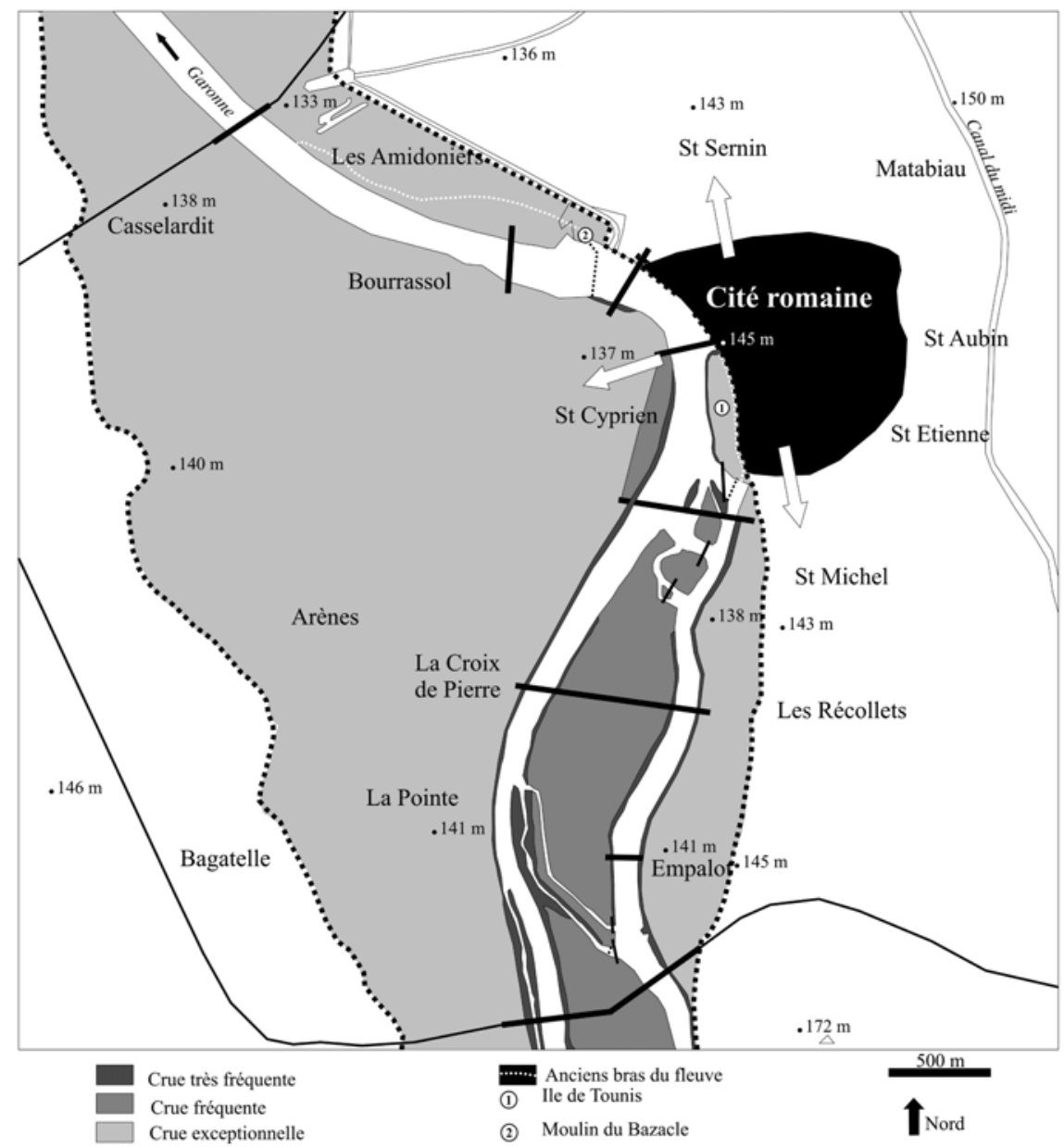

Figure 6. Les zones inondables à Toulouse.

Source: D'aprés DREAL, 2010, modifié.

louse, en raison du tracé du fleuve qui vient longer la rive droite. Une inondation détruit les ponts, les chaussées, les maisons et peut parfois être meurtrière. Les chroniques ont gardé en mémoire de nombreuses inondations catastrophiques.

La première mention d'inondation date du $\mathrm{V}^{\mathrm{e}}$ siècle, il s'agit de la grande crue postromaine de la Garonne, étudiée à travers les dépôts de matériaux (Astre, 1943). Cette crue aurait fait reculer une partie de la berge de rive droite dans les environs d'Empalot et modelé le tracé actuel de la Garonne et par la même favorisé le développement de la ville sur le site actuel. 
La chronique se précise au Moyen-Age surtout en aval de la ville, et reste incomplète pour la ville de Toulouse. L'analyse des archives du moulin du Bazacle devrait permettre de préciser les mentions des inondations depuis le XIIe siècle. Ce travail est encore à réaliser à Toulouse. En s'appuyant sur les travaux de Serret (1900), de nombreuses dates d'inondations peuvent être identifiées, sans que leur localisation ou leur ampleur puisse être précisée. Serret comptabilise 90 crues et inondations entre le VIe siècle et 1690. Il est difficile de vérifier et de préciser si ces inondations ont toutes touchées Toulouse. Néanmoins, parmi toutes les inondations reconnues, certaines ont affectées de manière significative la ville de Toulouse: le 11 mars 1281 (destruction d'un pont), 1430 (ruine de l'hôpital St Jacques), octobre 1435 (pont de pierre renversé), 30 mai 1486 (pont vieux emporté), 5 avril 1523 (pont vieux emporté et maisons de l'île de Tounis détruites), octobre 1536 (moulin du Bazacle détruit), 1589 (" plus grande inondation de mémoire d'homme "), 16 février 1599 (maisons de Tounis détruites et les murailles de St Cyprien renversées). Laffont (1997), quant à lui, qui a travaillé exclusivement sur Toulouse comptabilise 7 inondations pour le XIIIe siècle, 9 pour le XIVe siècle, 11 pour le XVe siècle, 4 pour le XVIe siècle et 10 inondations pour le XVIIe siècle. Pour le siècle des Lumières (de 1690 à 1790), il identifie 24 crues. Parmi ces dernières, il est possible d'évoquer : juin 1712 (chaussées endommagées, 50 maisons détruites à St Cyprien : "aiguat de San Barnabé "), 1727 (939 maisons détruites, 436 maisons endommagées), 2 mai 1765 (l'eau passe dans les trois lunes du Pont Neuf), 1770 (700 maisons détruites : "lou gran aïguat des rameoux"), 1772 (effondrement de l'Hospice St-Jacques, destruction de maisons, 50 victimes).

A partir du XIXe, l'utilisation de données quantitatives permet d'approcher plus précisément d'importance de ces événements. Parmi les plus importantes, il faut citer 1816 (hauteur de 5m10), 1827 (hauteur de 7m36), 30 mai 1835 (nombreuses victimes et 48 maisons détruites), juin 1855 (pont de pierre détruit), 1872 (hauteur de 5m06),.. Parmi tous ces événements, "lou gran aïguat de Sen Jean " du 23 juin 1875 est encore inscrit dans les mémoires toulousaines, où le faubourg St Cyprien est submergé par les eaux. L'inondation atteint une hauteur de $8 \mathrm{~m} 32$, plus haute crue mesurée à ce jour pour un débit estimé entre 6000 et $8000 \mathrm{~m}^{3} / \mathrm{s}$. Les dégâts à Toulouse sont considérables : 209 victimes, tous les ponts détruits, sauf le pont neuf, des centaines de maisons détruites.

De la fin du XIXe siècle jusqu'à aujourd'hui, les crues mesurées sont fiables. En ne retenant que les crues ayant dépassé la cote de $3 \mathrm{~m}$ (cote d'alerte fixé à $2 \mathrm{~m} 50$ pour un débit de $950 \mathrm{~m}^{3} / \mathrm{s}$ ), on peut comptabiliser 47 événements entre 1872 et aujourd'hui. Parmi les plus importantes, on peut citer 1905, 1910, 1952, 1977 et 2000.

La longue litanie des crues et des inondations ayant touché la ville de Toulouse permet de comprendre que la rive gauche n'est pas un site favorable à l'installation des hommes. Malgré ce fléau très présent à Toulouse, les toulousains ripostent et mettent en œuvre des aménagements dont le but est la défense contre les eaux pour permettre une meilleure valorisation de la ressource fluviale. 


\section{Les campagnes d'évergétismes toulousaines : origine de la maîtrise du fleuve?}

Le terme d'évergétisme est surtout utilisé dans le cadre des civilisations anciennes grecque et romaine. Lamboley (1997) nous en propose une définition : "pratique sociale des riches particuliers qui participent de leurs deniers à l'embellissement de leur cité ou prennent en charge une partie de ses obligations financières (...) ". A Toulouse, la rive gauche et les territoires proches du fleuve bénéficient depuis toujours de campagnes d'évergétismes de la part des élites toulousaines situés sur la rive droite, à l'abri des inondations. La dissymétrie sociale de la ville se matérialise par cette volonté, parfois précoce, parfois plus longue, de la rive droite de protéger les basses terres du fléau de la Garonne, jusqu'au moment où protégés, certains de ces quartiers changent de statut social et rejoignent celui des élites toulousaines.

\subsection{Composer avec les contraintes du site (de l'époque romaine jusqu'à la fin du XVle siècle)}

De la période romaine jusqu'à la fin du XVI e siècle, les aménagements de la Garonne à Toulouse ne sont pas directement des actions de protection contre les crues et inondations. Une des premières campagnes d'évergétisme est menée pour construire le rempart de la ville et le premier fléau combattu, indirectement, par la ville est certainement l'érosion des berges de la rive droite. La question des remparts antiques de la ville de Toulouse est encore aujourd'hui à l'origine de vifs débats scientifiques (Pailler, 2002). Etait-il continu sur l'ensemble du front urbain face à la Garonne ? La question n'a pas connu de réponse définitive. En tous cas, on trouve encore des traces de cet ouvrage au niveau de la rue de la Garonnette, au sud de la ville antique dans l'Institut Catholique. Avec cet ouvrage, seule la rive droite est protégée des affouillements du fleuve. Plus tard, au Moyen-âge, les remparts de la ville dans le quartier St Cyprien jouent un second rôle de protection face aux crues et inondations. De nombreuses représentations de Toulouse à cette époque nous montrent ce quartier totalement encerclé de remparts, hormis dans les environs de la prairie des Filtres (cf figure 7). Le système défensif sur la Garonne intègre les hôpitaux de la rive gauche et les relie entre eux par un mur défensif. L'histoire de la construction de ce rempart sur la rive gauche est ponctuée de destructions et avaries liées au fléau garonnais (Tourrilhes, 1987). Sur la berge de rive droite, plus haute, les maisons se présentent " dépourvues de défense et entièrement ouvertes sur le fleuve " (Laffont, 1997). Les habitations sont directement construites sur le bord de l'eau, à la limite des hautes eaux annuelles (cf figure 2). "De loin en loin, pour permettre à la navigation de commerce de disposer d'espaces de manipulation, la rangée des maisons s'interrompt pour faire place à des grèves d'échouage ou ports parfois dallés " (Pelletier, 1990). Les nombreux ports de cette période "devaient se limiter à des esplanades (cale ou gravier) agrémentés de quelques pieux fichés dans le lit du fleuve" (Tourrilhes, 1987). 


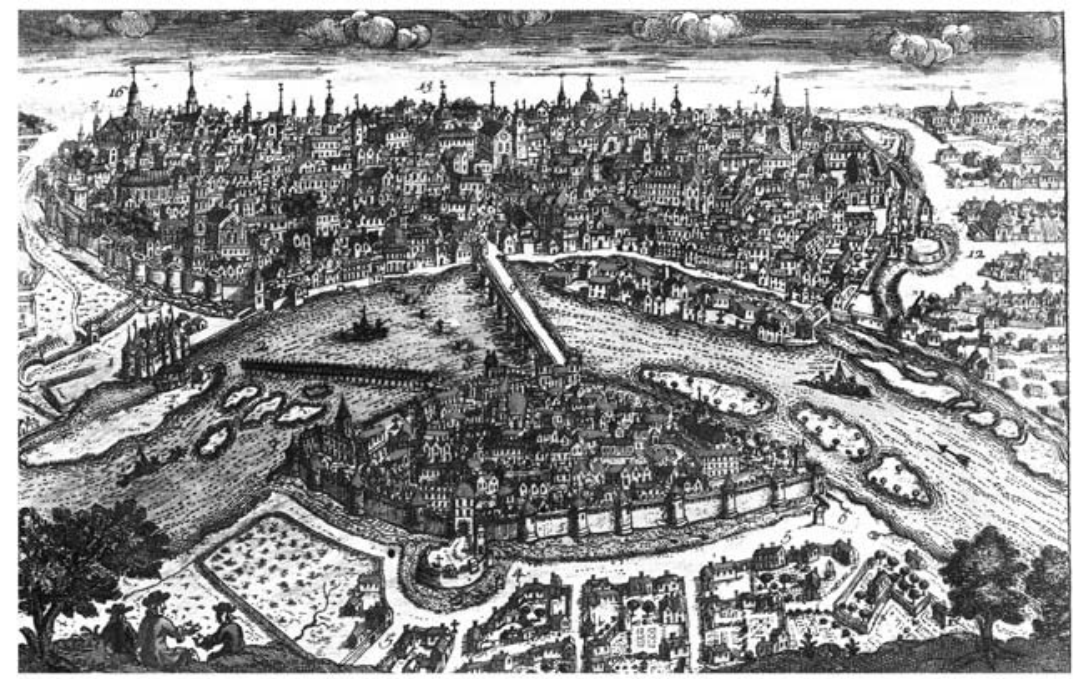

Figure 7. Les remparts de la ville comme protection face au fleuve.

Source: Milieu XVIle siècle, Archives Municipales de Toulouse, 20 Fi 304.

Il faut ajouter aux remparts, l'aménagement de ponts mais aussi de chaussées pour les moulins. "Aucun pont de Toulouse n'a résisté à la force des eaux avant la construction du Pont Neuf commencée en 1543 sous le règne de François $1^{\mathrm{e}}$ et inauguré en 1660 par Louis XIV " (Fortuné, 1988). L'histoire de la construction des ponts permet de voir en filigrane l'histoire des crues et inondations. Le pont romain et les nombreux ponts en bois médiévaux ont été emportés, reconstruits, détruits à nouveau. Par exemple le pont "viel " a été reconstruit cinq fois entre 1258 et 1414. De la même manière, le pont de la Daurade a été reconstruit à quatre reprises entre 1258 et 1414 . Tous ces témoignages de construction, destruction et reconstruction nous montrent une société encore très soumise aux caprices du fleuve garonnais. Durant la même période, la construction de chaussées viennent barrer le cours du fleuve. Elles sont liées au fonctionnement des moulins à eau dont le rôle économique a été très important pour la ville de Toulouse. Là aussi, l'histoire de la construction des chaussées des moulins toulousains est fortement liée à la fréquence des inondations qui détruisent régulièrement les ouvrages. Durant le Moyen Age, trois chaussées sont construites au niveau du quartier St Michel, dans les environs de la Daurade et du Bazacle (cf figure 8). Cette dernière construite à la fin du XIIe siècle est oblique (longueur d'environ 500 m) et s'étend de l'Hôtel Dieu jusqu'au moulin lui-même et deviendra perpendiculaire au fleuve quelques siècles plus tard.

L'aménagement des remparts, des ponts, des chaussées ont pour conséquence de perturber l'écoulement du fleuve, et ces ouvrages sont fréquemment détruits par les aïguats. A partir du XVIIe siècle les campagnes d'évergétismes toulousaines montrent une vraie volonté de maîtriser et fixer le fleuve. 


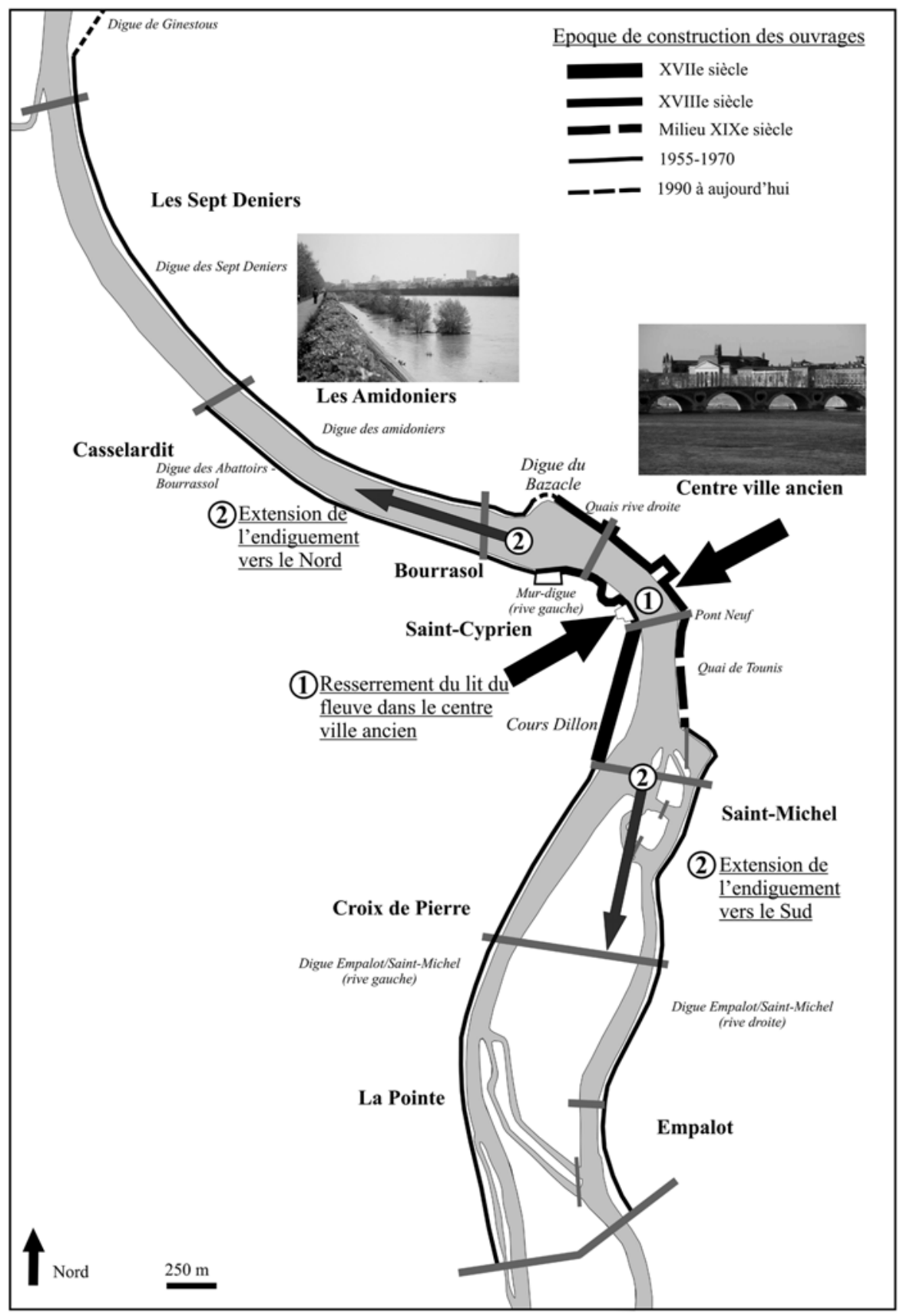

Figure 8. Les aménagements de défense contre les inondations à Toulouse. 


\subsection{Une maîtrise partielle et un embellissement du front fluvial (XVIle siècle jusqu'à la seconde moitié du XXe siècle)}

A partir du XVII ${ }^{\mathrm{e}}$ siècle, la ville de Toulouse tente de fixer et de maîtriser la Garonne, afin d'échapper au moins en partie à ses débordements. Plusieurs aménagements de cette période sont encore visibles aujourd'hui. Le premier aménagement d'envergure pour lutter contre les crues et les inondations date de 1601 et correspond à l'aménagement d'un remblai sur $540 \mathrm{~m}$ de longueur, dont le but est de protéger le faubourg St-Cyprien des envahissements des eaux (Fortuné, 1988). Ce remblai forme alors la digue du Cours des Ormes. C'est aussi en 1660 que la construction du Pont Neuf se termine et ce pont restera pendant très longtemps le seul pont sur la Garonne. Vers la fin du XVIIe siècle et le début du XVIIIe siècle, les établissements d'assistance et de renfermement des pauvres de St Cyprien sont bâtis comme l'Hôtel Dieu St jacques et l'Hôpital général St Joseph de la Grave (Taillefer, 2005).

D'autres travaux sur les berges tendent à rétrécir le lit de la Garonne, c'est le cas avec la création de l'aile amont de l'Hôtel Dieu en aval du pont neuf. C'est aussi le cas sur la rive droite avec " les usurpations des pères bénédictins de la Daurade (...) qui avaient formés au moyen de pilotis plantés dans le fleuve, un jardin qui bouchait complètement la première arche du Pont neuf et en partie la seconde " (Laffont, 1997). Tous ces aménagements créent de véritables conflits entre bénédictins, propriétaires de moulins, municipalité et riverains.

D'autres travaux de cette époque montrent un certain découragement de la part de la ville. Sur la rive droite au niveau de la pointe sud de l'île de Tounis, en 1624, "les inondations de la rivière commencèrent d'emporter une partie desdites maisons (...)" (Archives Municipales de Toulouse, DD 142). Consécutivement à ce désastre une petite digue est construite et terminée en 1633, mais cet ouvrage ne résiste pas à la crue de 1635. On reconstruit en 1639 une digue qui est à nouveau détruite en 1667 (Laffont, 1997). "Il fut résolu de faire un quay qui fut suffisant pour remparer l'isle et détourner le cours de la rivière " (Archives Municipales de Toulouse, BB 281). Les travaux sont entrepris puis sont arrêtés à cause d'une série d'inondations $(1670,1672)$ et de conflits entre la ville et les entrepreneurs. A la fin du XVIIe siècle, le projet de quai est abandonné et l'île se trouve comme au début de ce siècle sans défenses.

La digue du Cours des Ormes, les piles du Pont Neuf, les bâtiments s'avançant sur le fleuve et les chaussées ont pour conséquence de modifier durablement les écoulements du fleuve, ce qui se traduit par des érosions sur la rive droite et des atterrissements sur la rive gauche. "Le XVIIIe siècle est vraiment celui des grands travaux d'aménagement et de protection de la ville contre la Garonne "(Fortuné, 1988). C'est surtout durant la seconde moitié du XVIIIe siècle que ces travaux sont exécutés. Durant cette période, les quais construits sur la rive droite et la rive gauche ont une double fonction à la fois pour la navigation mais aussi pour canaliser les eaux de crues à l'intérieur 
du lit mineur. La berge de la rive droite de l'île de Tounis jusqu'au Bazacle se trouve aménagée par des quais en brique rouge, dans lesquels sont insérés les ports du Vidou et de la Daurade. La rive droite entre le pont neuf et le Bazacle est réalisée entre 1741 et 1789 (cf figure 8). Il faudra attendre le XIXe siècle pour voir les travaux se prolonger et se terminer sur l'île de Tounis (1850-1866). La rive gauche connaît la même dynamique puisque la digue des Ormes est remplacée par une digue en briques rouge au niveau du Pont Neuf et prolongée jusqu'au niveau de la porte de Muret. L'ancien cours des ormes subissait l'assaut continuel de la Garonne consécutif à la reconstruction et au rehaussement de la chaussée du Bazacle en 1713, détruite en 1709 par une crue de débâcle (fonte des glaces). La chaussée du moulin du Château Narbonnais provoque, elle aussi, des vulnérabilités accrues du quartier St Michel face aux inondations. Contrairement, au faubourg de St Cyprien, celui de St Michel n'est pas protégé. L'ancien cours des Ormes devient le Cours Dillon construit entre 1758 et 1762. Il est planté de quatre rangées d'Ormes. Ces arbres lui confèrent une fonction de promenade non loin du faubourg St Cyprien.

Tous ces aménagements ont été conçus pour protéger la ville mais aussi pour l'embellir. Saget, directeur des travaux publics de la province, fait édifier des maisons audessus des quais, en reprenant les mêmes matériaux. Les façades des maisons de la rive droite sont rythmées par de grandes arcades se répétant de la place St-Pierre au port de La Daurade. Cet alignement de quais et d'habitations est devenu un des paysages identitaires de la ville de Toulouse. Il faut ajouter à tous ces aménagements la construction du canal de Brienne en 1768, qui permet la liaison entre le canal du Midi et la Garonne en amont du Bazacle. Par conséquent, le XVIII e et du XIXe siècle est un siècle phare pour la ville de Toulouse et les paysages du front urbain historique sont directement hérités de cette période.

\subsection{Contraindre la Garonne (seconde moitié du XXe siècle à aujourd'hui)}

La fin du XIXe siècle et le début du XXe siècle est surtout marqué par la mutation des usages : l'abandon de la navigation et l'arrivée de l'hydroélectricité. Les anciens moulins, comme le Bazacle, sont transformés en usine hydroélectrique ou alors sont détruits comme le moulin du Château du Narbonnais. La seconde moitié du XXe siècle voit une explosion urbaine qui oblige la société riveraine à prolonger les digues du XVIII ${ }^{\mathrm{e}}$ siècle (cf figure 8). A la fin des années 1960, "le lit de la Garonne a été enfermé dans un système de digues en béton depuis les ponts d'Empalot, jusqu'au pont de Blagnac " (CUIET, 1993). Ces travaux de défense dotent la ville d'une protection par rapport aux crues et inondations de type de celle de 1875. Cependant, les matériaux (béton) et la hauteur des digues viennent rompre la dynamique et les aménagements du XVIII ${ }^{\mathrm{e}}$ siècle. Les nouveaux travaux témoignent d'un rapport ville/Garonne, où le fleuve suscite peu d'intérêts si ce n'est de le contraindre et le maîtriser totalement. Les anciens 
canaux de fuite du moulin du Château du Narbonnais et du moulin du Bazacle sont comblés. Ainsi, en 1950 la Garonnette et le canalet sont asséchés. "Toulouse géographiquement et historiquement doit son existence à la Garonne, pourtant peu à peu elle lui a tourné le dos"(CUIET, 1993). La voie d'eau a été remplacée progressivement par le chemin de fer puis par la route. C'est durant le XX $\mathrm{X}^{\mathrm{e}}$ siècle que la ville de Toulouse rejette sur les îles du Ramier tous les aménagements ne trouvant pas leur place ailleurs comme la poudrerie, l'incinérateur d'ordures ménagères, les bâtiments de foires, le stade... De plus, de nombreux parkings sont aménagés sur les berges comme au port de la Daurade ou St-Cyprien par exemple.

Depuis quelques années, une prise de conscience collective a permis à la ville de Toulouse de renouer des liens avec son fleuve.

\subsection{Le front fluvial : la parure de la ville (années 1990 à aujourd'hui)}

Depuis les années 1990, des travaux de protection sont réalisés dans la ville (surélévation du Cours Dillon, aménagement de stations de relevage des eaux pluviales, aménagement de portes métalliques,...). Tous ces aménagements correspondent à des retouches du système d'endiguement mis en place dans les périodes précédentes (cf figure 8). Néanmoins, des digues sont construites comme la digue de Ginestous, celle du Bazacle. Ces ouvrages, outre leur fonction de protection, montrent un effort d'intégration au paysage puisqu'elles font l'objet de tentatives de végétalisation.

Durant la même période, la municipalité met en place un plan Garonne dont " les objectifs sont la valorisation des paysages, la protection des équilibres naturels et la mise en place d'espaces verts " (CUIET, 1993). Ainsi, la mise en valeur du fleuve passe par le développement de pôles de loisirs et de détente, la redécouverte de la nature en ville, mais aussi par la mise en valeur du patrimoine architectural et la reconversion d'édifices importants. Partout, des lieux de promenades sont " aménagés " sur les berges du fleuve. La promenade Henri-Martin permet le cheminement de la place St-Pierre au Port Garaud. La prairie des Filtres, ancien terrain vague a été transformé en jardin. Au sud, un cheminement permet la promenade dans les environs d'Empalot. Ailleurs, un jardin met en valeur le rempart médiéval entre les allées Charles de Fitte et la Garonne. Au total, ce sont 16 ha de parcs et de promenades qui ont été aménagés sur les berges de la Garonne.

De plus, le fleuve lui-même s'anime avec la réalisation d'aménagements pour les sports nautiques comme l'Aviron ou le Canoë-Kayak. Le port de la Daurade a vu l'arrimage d'un bateau restaurant. Toutes ces activités permettent au fleuve de s'animer à nouveau.

Enfin, de nombreux bâtiments ont été réhabilités et redonnent un certain dynamisme aux berges du fleuve. Ainsi, plusieurs lieux, après réhabilitation ont changé d'usage. La 
galerie du château d'eau et le Théâtre Garonne ont été aménagés dans des bâtiments voués à l'abandon. L'ancienne manufacture des Tabacs est devenue une université. "L'espace Bazacle ", salle d'exposition, remplace l'usine électrique. Le musée d'art contemporain a été aménagé à l'intérieur des anciens abattoirs de la ville. Des spectacles en plein air s'organisent au port de la Daurade. Aujourd'hui, beaucoup d'initiatives s'appuient sur la mise en valeur architecturale et culturelle des berges de la ville (cf figure 9).

Début XXe siécle

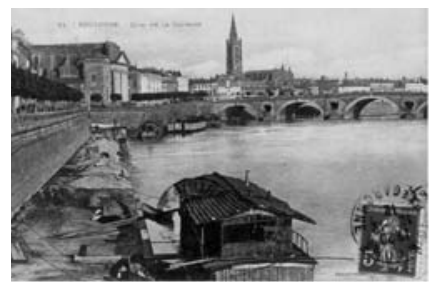

Avril 2011 (Ph Valette)

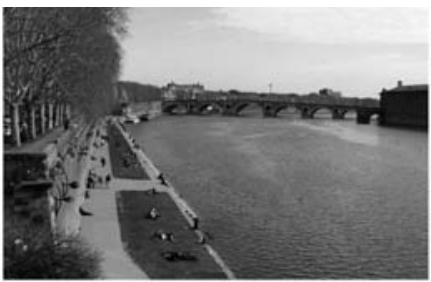

Mai 2012 (Ph Valette)

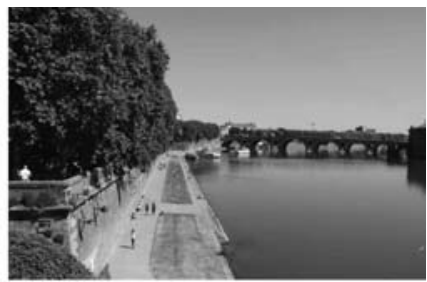

La Garonne dans le centre ancien de Toulouse (Pont Neuf) a fait l'objet de nombreuses valorisations ponctuelles.

Début XXe siécle

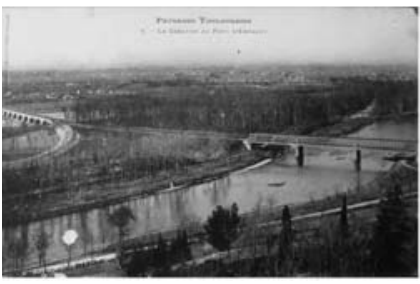

Février 2011 (Ph Valette)

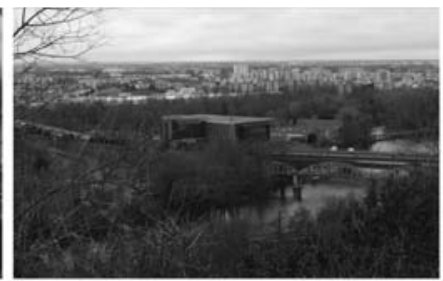

Juin 2013 (Ph Valette)

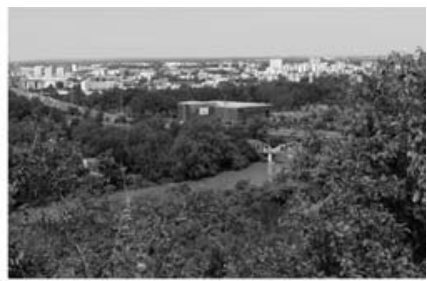

La Garonne aux limites sud de la ville (périphérique): I'urbanisation s'étend dans la plaine inondable et les bords du fleuve restent assez peu valorisés.

Figure 9. Observer les paysages fluviaux urbains toulousains.

Cependant, cette valorisation reste ponctuelle et n'a pas l'envergure du renouveau de la Garonne à Bordeaux par exemple. L'ambition de la nouvelle équipe municipale est de remettre la Garonne au cour de la ville. Depuis quelques années, le projet de Grand parc Garonne a été défini où il s'agit de reconquérir et aménager $32 \mathrm{~km}$ de berges de la Garonne. Ici, le territoire déborde largement le seul centre-ville ancien puisque ce projet concerne la confluence de l'Ariège jusqu'aux environs de St Jory avec comme objectif de relier les espaces verts entre eux à partir de circulations douces (promenade à pied, vélo). Parmi les projets phares nous pouvons citer la destruction d'une partie du parc des expositions pour reconstituer l'ancien parc toulousain (espace vert détruit au cours du XXe siècle par l'urbanisation). Peu de réalisations concrètes ont été réalisées de nos jours. 


\section{Conclusion}

La ville de Toulouse s'est largement et durablement développée sur les berges de la Garonne. Les berges du fleuve apparaissent alors comme une interface entre le fleuve et la ville, où transparaissent les politiques de défense contre les eaux d'inondation, les aménagements de valorisation de la ressource hydrique... Les paysages fluviaux urbains toulousains actuels sont une matérialisation des systèmes de lutte contre les crues et inondations du fleuve en grande partie hérité des politiques volontaristes du XVIIIe et du XXe siècle.

Aujourd'hui, la Garonne à Toulouse est un décor et le fleuve fait, de plus en plus, l'objet d'aménagements visant à améliorer son aspect esthétique et paysager. Le souci de la qualité des paysages s'est traduit par l'aménagement d'espaces verts et une mise en valeur architecturale au sein du front urbain. Ce souci d'intégration des aménagements dans le paysage se traduit, par exemple, par la plantation de vigne vierge sur les digues en béton de la seconde moitié du $\mathrm{XX}^{\mathrm{e}}$ siècle.

La connaissance et la détermination des différentes temporalités dans la construction des paysages fluviaux urbains toulousains, mis en avant dans ce travail, nous permettent, également de replacer les projets actuels dans ce contexte historique. Face aux évolutions futures liées au projet du Grand parc Garonne, nous avons mis en place depuis deux ans un observatoire des paysages de la Garonne, où les sites d'observation choisis à Toulouse s'appuient sur cette connaissance géohistorique (http://w3.geode. univ-tlse2.fr/obs_paysage.php et figure 8).

D'autre part, si le renouveau Ville / Fleuve est bien réel sur le front fluvial toulousain ancien, où les aménagements malgré leur mutation d'usage structurent durablement les paysages, qu'en est-il pour les espaces péri-urbains en amont et en aval de la ville ? Le projet actuel prévoit de valoriser ces territoires, aujourd'hui largement délaissés. Entre le désir de fleuve et danger, l'enjeu de la relation de la ville étendue au fleuve pourrait alors se jouer sur ses marges.

\section{Bibliographie}

Armengaud R., Vieu H. (sous la direction de) (1998) Un quartier de Toulouse, d'Empalot à Port-Garaud. Au cours des siècles, au fil de l'eau, Mémoires des pays d'Oc, 180 p.

Arramond J.C., Requi C., Vidal M., 2004, La Toulouse des Volques Tectosages (Vieille-
Toulouse, Estarac, St-Roch), in Guide de l'exposition Gaulois des pays de Garonne (IIe-Ier siècle avant J-C), 92 p, pp 42-50.

Arrouy J.M., 2005, L'île de Tounis, Histoire d'un quartier an coeur de Toulouse (du XVIIe au XXe siècle), Loubatières, $187 \mathrm{p}$. 
Astre G., 1943, "La grande crue post-romaine de la Garonne ", Bulletin de la Société d'Histoire Naturelle de Toulouse, Tome 78.

Bordes F. (sous la direction de), 2005, Toulouse parcelles de mémoire, 2000 ans d'bistoire urbaine au regard de huit siècles d'archives municipales, $381 \mathrm{p}$.

Cathala Y., 1962, Les épidémies de choléra à Toulouse au XIXe siècle, Toulouse, Veuve Lion, $215 \mathrm{p}$.

Chalande J., 1919, Histoires des rues de Toulouse. Monuments, institutions, habitants, Toulouse, 3 tomes.

Centre Urbain d'Initiation à l'Environnement de Toulouse (CUIET), 1993, La vie au bord du fleuve, $72 \mathrm{p}$.

Coppolani J., 1984, Toulouse, étude de géographie urbaine, ouvrage publié avec le concours du Ministère de l'Education Nationale, Privat.

Dubourg M.A., 1883, Histoire du Grand Prieuré et des diverses possessions de l'ordre de St Jean de Jérusalem dans le Sud-Ouest de la France, Toulouse, pp 40-41.

Fortuné M., 1988, Usages passés et écologie de la Garonne, Thèse de Doctorat en écologie, Université Paul Sabatier, 162 p.

Labrousse M., 1968, Toulouse antique, Ed. de Broccard.

Lafaille G. de, 1687, Annales de la ville de Toulouse depuis la réunion du comté de Toulouse à la couronne, avec un abrégé de l'ancienne histoire de cette ville et un recueil de divers titres et actes pour servir de preuves ou d'éclaircissements à ces Annale, Toulouse, G.-L. Colomiez, 2 t., 1687-1701

Laffont J.L., 1997, Policer la ville. Toulouse, capitale provinciale au siècle des Lumières, Thèse de doctorat en Histoire, Université de Toulouse Le Mirail.

Lambert R., 1982, "Les crues de la Garonne ", in Ferro M. (Sous la direction de ...), Une bistoire de la Garonne, Collection des fleuves et des hommes, Ramsay, Paris, p. 42-81.

Lamboley J.L., 1997, Lexique d'histoire et de civilisation romaines, Ellipse Marketing, $381 \mathrm{p}$.

Loncan B., 1998, Les quartiers de Toulouse. L'île du ramier, Itinéraires du Patrimoine, $\mathrm{n}^{\circ}$ 176, Accords Editions, 20 p.

Macé L., "Toulouse médiévale ( $12^{\mathrm{e}}-13^{\mathrm{e}}$ siècles), in Bordes F. (sous la direction de), 2005, Toulouse parcelles de mémoire, 2000 ans d'histoire urbaine au regard de huit siècles d'archives municipales, 381 p., pp. 71-76.

Marconis R., 1996, "Toulouse ", in Pinol J.L., Atlas historique des villes de France, Hachette, 318 p., pp. 232-255.

Mercié P., 2007, Légendaires moulins à eau aujourd'hui disparus. Du triangle rose Toulouse, Montauban, Albi, 367 p.

Pailler J.M. (sous la direction de), 2002 ; Tolosa. Nouvelles recherches sur Toulouse et son territoire dans l'Antiquité, Ecole Française de Rome, 601 p.

Pelletier J., 1990, "Sur les relations de la ville et des cours d'eau ", Revue de Géographie de Lyon, vol. 65, p. 233-239.

Peyrusse L., " Des Lumières aux percées haussmaniennes : les métamorphoses d'une ville, in Bordes F. (sous la direction de), 2005, Toulouse parcelles de mémoire, 2000 ans d'histoire urbaine au regard de huit siècles d'archives municipales, 381 p., pp. 223-226.

Serret J., 1900, Les débordements de la Garonne et de ses affluents depuis les temps anciens jusqu'à nos jours, $97 \mathrm{p}$.

Syndicat Mixte d'Etude et de Programmation pour l'Aménagement de la Garonne (SMEPAG), 1989, Schéma de protection contre les eaux de Garonne, Tome 1, Monographies des crues de la Garonne (du Pont du Roy au Bec d'Ambès), $168 \mathrm{p}$. 
Syndicat Mixte d'Etude et de Programmation pour l'Aménagement de la Garonne (SMEPAG), 1989, Schéma de protection contre les eaux de Garonne, Tome 3, 47 p.

Taillefer, M. (2005) “ L'Ancien Régime (16e-18e siècle), les prémices des grandes mutations ", in Bordes F. (sous la direction de), 2005, Toulouse parcelles de mémoire, 2000 ans d'histoire urbaine au regard de huit siècles d'archives municipales, 381 p., pp. 157-162.

Tourrilhes, V. (1987) La Garonne à Toulouse au Moyen-Age. Etat de la question, mémoire de maîtrise, Université de Toulouse Le Mirail, 200 p.

Vaginay, M., "Toulouse antique ", in Bordes F. (sous la direction de) (2005) Toulouse parcelles de mémoire, 2000 ans d'histoire ur- baine au regard de buit siècles d'archives municipales, 381 p, pp 23-28.

Valette, P., Carozza, J-M. (2011) " Les paysages fluviaux urbains : évolution du rapport ville/fleuve à travers l'exemple de l'agglomération toulousaine et la Garonne ", in Revue du Nord, Zones humides et villes d'hier et d'aujourd'hui : des premières cités aux fronts d'eau contemporains, Hors-série, $\mathrm{N}^{\circ} 26$, pp. 219-237.

Valette, P. (2002) Les paysages de la Garonne: les métamorphoses d'un fleuve (entre Toulouse et Castets-en-Dorthe), Doctorat de Géographie, Université Toulouse Le Mirail, $554 \mathrm{p}$.

Wolff, P. (sous la direction de ...) (1974) Histoire de Toulouse, éd. Privat, 552 p. 
